\title{
Study of Ascitic Fluid Micro RNA-155 in Patients with Spontaneous Bacterial Peritonitis
}

\author{
F.M.Abd Al-Salam ${ }^{1}$, N.E.El-Toukhy ${ }^{1}$, A.A.Mohamed ${ }^{2}$, and A.S.Sultan ${ }^{1}$ \\ ${ }^{1}$ Hepatology, Gastroenterology and Infectious Diseases, Dept., Faculty of Medicine, Benha Univ., Benha, Egypt \\ ${ }^{2}$ Biochemistry and Molecular Biology, National Hepatology Tropical Medicine Research Institute \\ E-mail: A.S.Sultan2@gmail.com
}

\begin{abstract}
Background: In patients with cirrhosis, spontaneous bacterial peritonitis is the most common bacterial infection. This research aims at studying the ascititc fluid Micro RNA-155 as a possible biomarker in spontaneous peritonitis. Method: 187 liver cirrhosis and ascites were chosen, 97 SBP patients and 90 SBP patients, all patients had historical history, thorough and local examination, comprehensive investigations, abdominal ultrasonography, diagnostic abdominal paracentesis and Micro RNA-155 evaluation. Results: in SBP, the average value of TLC, Polymorphene, and RNA-155 was considerably greater than non SBP, but in the SBP group the average value of ascetic albumin, glucose, and protein was considerably lower than in the non-SBP group. For MiR-155, the optimal balance of sensitivity and specificity was 0.022 for ROC as a marker with sensitivity 60 percent and specificity 62.0 percent and surface area under curve 0.639. No statistically significant link exists among SBP patients among Micro RNA-155 with TLC, polymorphs, MELD score and Child Pugh score values. Conclusion; Although MiR-155 was increased in the ascetic fluid of SBP patients, and is a predictor for SBP development, it cannot be employed as diagnostic marker in the SBP, according to this research.
\end{abstract}

Keywords: Ascites, Micro RNA-155, Spontaneous Bacterial Peritonitis, SBP.

\section{Introduction}

The life-threatening consequence of cirrhosis and ascites, Spontaneous Bacterial Peritonitis (SBP) is linked with much greater mortality and may be difficult to diagnose since the clinical presentation is greatly varied and ascite cultures seldom are positive. The current clinical standard of diagnosing SBP is the count of ascites neutrophil (ANC) at 250 cells $/ \mathrm{mm} 3$ for peritonitis [1].

The death risk is $80 \%$ higher in cirrhosis patients who develop due to spontaneous bacterial peritonitis septic shock. In addition, the hospital mortality rate rises by 1,86 times per hour of delay in antimicrobial therapy [2].

The microRNA (miR) was characterised as tiny, non-coding nucleotide RNA of around 21-25[3].

They control gene expression by binding to messenger RNA, and are involved, including liver disease and inflammation, in key biological processes. Moreover, they are considered as possible biomarkers for a number of disorders including liver illnesses because of their size and capacity to circulate [4]. While serum levels of miRs are recognised biomarkers for liver disease, their concentration and potential significance as biomarkers for other biofluids such as ascites in cirrhosis remain uncertain.

The macrophage activation involves MiR-155, which is the major cell population in ascites with lymphocytes [5].

Expression of MiR-155 increased in peritoneal immune cells after a bacterial challenge in a mouse peritonitis model and after intraperitoneal Lipopolysaccarid. Furthermore, miR-155 was demonstrated to be necessary for antibacterial protection. In patients with spontaneous bacterial peritonitis, miR-155 was also elevated [4].
This research was targeted at studying ascititc fluid RNA-155 in spontaneous bacterial peritonitis as a possible biomarker.

\section{Patents and methods}

In 187 patients hospitalized to the Department of Hepatology, Gastroenterology and Infectious Diseases at the Benha University Hospital and the Ahmed Maher Teaching Hospital, this cross-sectional research was conducted on a basis of two groups based on the presence or absence of SBP.

- Group I: Includes 97 patients with cirrhosis and ascites and SBP.

- Group II: Includes 90 patients with cirrhosis and ascites without SBP.

\subsection{Inclusion criteria}

Adult patients with cirrhotic ascites.

\subsection{Exclusion criteria}

- Secondary bacterial peritonitis

- Patients with mycobacterial or fungal peritonitis .

- Evidence of gastrointestinal bleeding or bacterial infection in the preceding 6 weeks.

- Treatment with non-absorbable antibiotic in the preceding 6 weeks.

- Other non-peritoneal infection (skin infection, chest infection, biliary tract infection, urinary tract infection, gastroenteritis, dental infection, meningitis).

All patients were subjected to thorough history taking, Full general and local examination, Full investigations, Abdominal ultrasonography, and diagnostic abdominal paracentesis, and Micro RNA155 level. 


\subsection{Statistical analysis}

Data collected were reviewed, coding and statistical analysis of collected data were done by using SPSS program (statistical package of social science; SPSS Inc., Chicago, IL, USA) version 24 for Microsoft Windows. A-Descriptive statistics: Mean and standard deviation $($ mean \pm SD) were calculated to measure central tendency and dispersion of quantitative data. Frequency of occurrence was calculated to measure qualitative data. B-Analytic Statistics: Comparing groups was done using : Chi-square-test $(\chi 2)$ :for comparison of qualitative data, Student $t$ test to determine the significance in the difference between two means of parametric data, Mann-Whitney $U$ test to determine the significance in the difference between two variables of non parametric data, Pearson and Spearman's Rho correlation coefficients were calculated for association between parametric and non parametric data respectively, Receiver Operating Characteristics (ROC) curve was done to estimate the best cut off point then calculation of sensitivity, specificity with their $95 \%$ confidence interval was done.

\section{Results}

The mean age was statistically higher in patients with SBP than in patients without SBP. There is no statistically difference between two groups, as regards to gender. table (1)

The mean value of TLC, polymorphs and micro RNA-155 was significantly higher in SBP group than non SBP group, while the mean value of ascetic albumin, glucose and protein was significantly lower in SBP group than in non SBP group, no statistical significant difference between the two groups as regards to SAAG. table (2)

There is a no statistically significant correlation between Micro RNA-155 with TLC, Polymorphs, MELD score and Child Pugh score values among patients with SBP. table (3)

Table (1) Demographic features of the studied patients.

\begin{tabular}{|c|c|c|c|c|c|}
\hline \multirow{3}{*}{$\begin{array}{l}\text { Socio-demographic Data } \\
\text { Age (mean } \pm \text { SD) } \\
\text { Gender }\end{array}$} & \multirow{2}{*}{\multicolumn{2}{|c|}{ 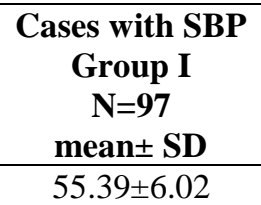 }} & \multicolumn{2}{|c|}{$\begin{array}{c}\text { Cases without SBP } \\
\text { Group II } \\
\mathrm{N}=90 \\
\text { mean } \pm \text { SD } \\
\end{array}$} & \multirow[t]{2}{*}{$\begin{array}{l}\text { P value } \\
<0.001 *\end{array}$} \\
\hline & & & & .42 & \\
\hline & $\mathrm{N}$ & $(\%)$ & $\mathrm{N}$ & $(\%)$ & \\
\hline Male & 51 & 46 & 54 & 60 & \\
\hline Female & 52.6 & 47.4 & 36 & 40 & 0.307 \\
\hline
\end{tabular}

SD = Standard deviation $\quad$ SBP $=$ spontaneous bacterial peritonitis

Table (2) Ascitic fluid analysis in studied patients

\begin{tabular}{|c|c|c|c|}
\hline Ascitic Fluid Analysis & $\begin{array}{c}\text { Cases with SBP } \\
\text { Group I } \\
\mathrm{N}=97 \\
\text { mean } \pm \text { SD } \\
\end{array}$ & $\begin{array}{c}\text { Cases without SBP } \\
\text { Group II } \\
\mathrm{N}=90 \\
\text { mean } \pm \text { SD } \\
\end{array}$ & $\mathrm{P}$ value \\
\hline Total leucocyte count $\left(10^{3} / u l\right)$ & $3428 \pm 3234.8$ & $878.6 \pm 2098.7$ & $<0.001 *$ \\
\hline Polymorphs $\left(10^{3} / \mathrm{ul}\right)$ & $705.09 \pm 606.29$ & $91.84 \pm 23.96$ & 0.008* \\
\hline Ascitic Albumin (g/dL) & $0.53 \pm 0.29$ & $0.63 \pm 0.23$ & $0.016 *$ \\
\hline Protein $(g / d L)$ & & & $<0.001 *$ \\
\hline Ascitic glucose (g/dL) & $\begin{array}{c}2.08 \pm 0.45 \\
48.96 \pm 6.19\end{array}$ & $\begin{array}{c}2.40 \pm 0.96 \\
81.61 \pm 67.03\end{array}$ & $<0.001 *$ \\
\hline SAAG (g/dL) & $2.27 \pm 0.86$ & $2.64 \pm 3.01$ & 0.237 \\
\hline MicroRNA-155 (CT) & $1.59 \pm 0.49$ & $1.37 \pm 0.48$ & $0.002 *$ \\
\hline
\end{tabular}

SAAG = serum ascetic albumin gradient $\mathrm{CT}=$ cycle threshold

Table (3) Correlation between Micro RNA-155 with some variables

\begin{tabular}{lcc}
\hline Correlation of Micro RNA-155 with: & Correlation Coefficient (r) & P value \\
\hline TLC & 0.037 & 0.616 \\
Polymorphs & -0.013 & 0.856 \\
MELD Score & -0.069 & 0.351 \\
Child Pugh Score & -0.040 & 0.583 \\
\hline
\end{tabular}

TLC $=$ Total leucocyte count

MELD =Model for end stage liver disease 
For Micro RNA-155 as a marker for SBP the optimum balance between sensitivity and specificity was at a cut - off value $0.022 \mathrm{CT}$ with sensitivity $60 \%$ and specificity $62.0 \%$ and area under the curve 0.639 . fig. (1).
For PMN as a marker for SBP the optimum balance between sensitivity and specificity was at a cut - off value $230\left(10^{3} / \mathrm{ul}\right)$ with sensitivity $100 \%$ and specificity $100 \%$ and area under the curve 1 . Fig. (2)

Micro RNA-155, TLC and serum creatinine are predictors for SBP with different variations. table (4)

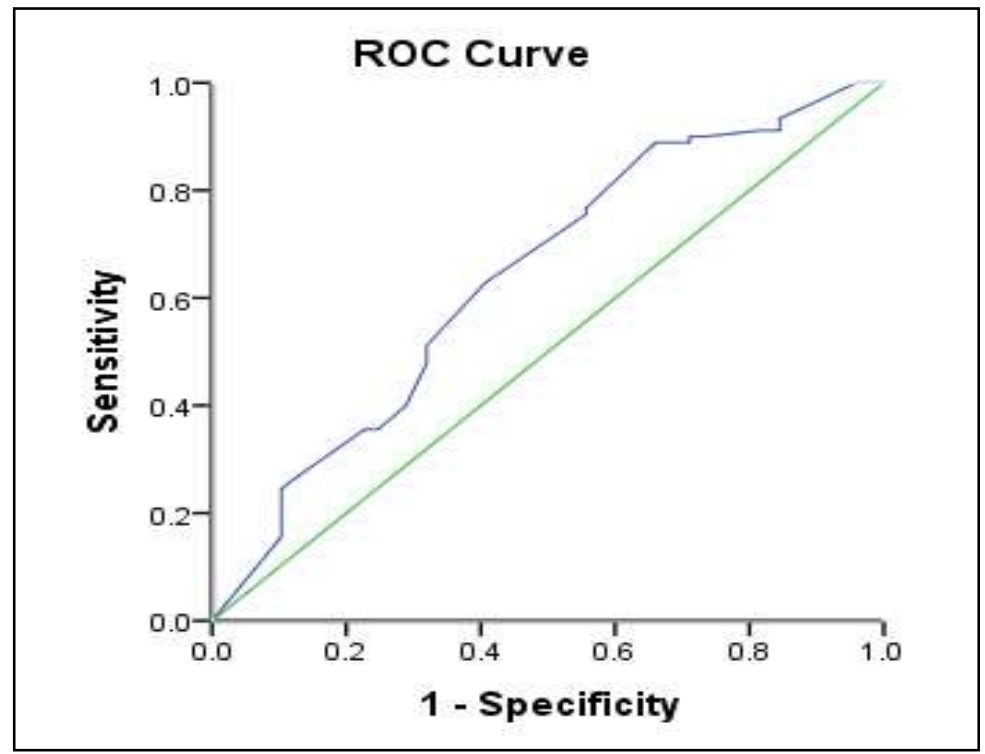

Fig. (1) ROC curve analysis for Micro RNA-155.

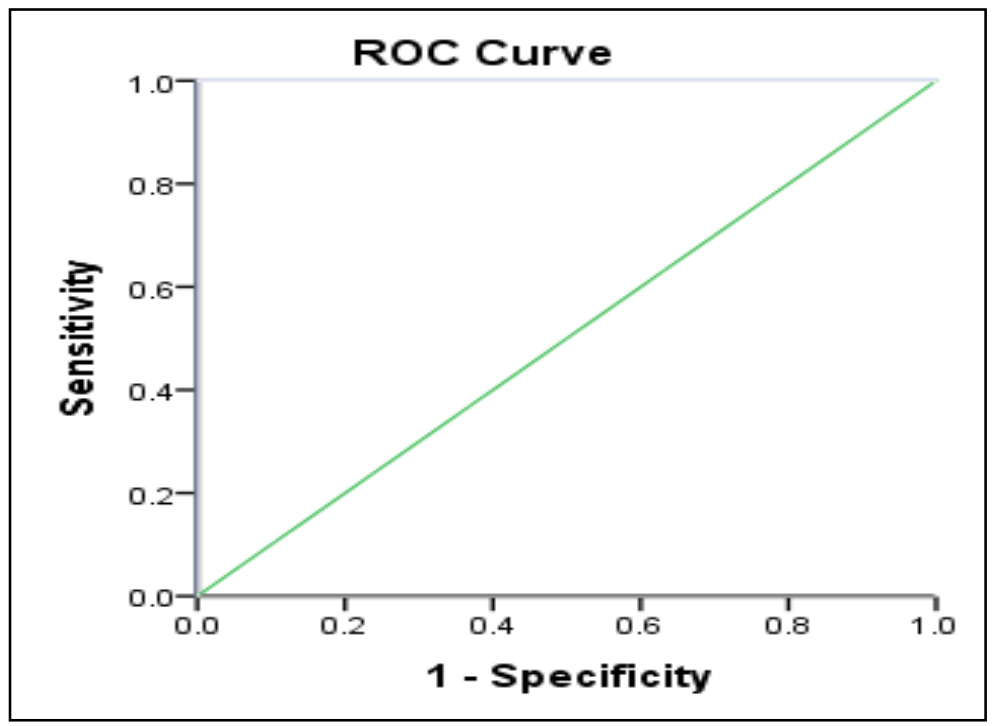

Fig (2): ROC curve analysis for PMN.

Table (4) Multivariate analysis for predictors of SBP.

\begin{tabular}{llll}
\hline & Beta coefficient & Standard error & p value \\
\hline & & & \\
Micro RNA-155 & 1.841 & .421 & $\mathbf{0 . 0 0 0 5 *}$ \\
TLC & 3.061 & .433 & $<\mathbf{0 . 0 0 1 *}$ \\
Creatinine & 1.461 & .410 & $<\mathbf{0 . 0 0 1 *}$ \\
\hline
\end{tabular}

SBP $=$ spontaneous bacterial peritonitis $\quad$ TLC $=$ Total leucocyte count 


\section{Discussion}

As far as the ascetic fluid analysis is concerned, the results in this study have found that in SBP patients the total number of leukocytes in ascetic fluid was significantly higher than in patients without SBP, such results are consistently consistent with Yildiri et al.[6] and Giron-Gonzzales et al.[7] which reported higher ascetic fluid TLC in SBP patients than patients without SBP.

With respect to ascetic fluid polymorphonuclear leukocytes, the results showed that in patients with SBP group the PMN count was significantly higher than in non-SBP groups, these findings are consistent with Nabiel et al.,[8] and El-Toukhy and Emam,[9] which reported higher ascetic fluid PMN in SBP patients compared with non-SBP patients.

As for ascetic fluid-total protein levels, this is consistent with Nabiel et al., [8] and Paul et al.[10] who have reported that ascitic fluid opsonic activity closely correlates with fluid protein concentration; fluids $<1.0 \mathrm{~g} / \mathrm{dl}$ of protein have reported decreasing opsonic activity and thus highly susceptible to protein concentrations

With respect to the amount of ascetic fluid albumin in SBP patients, this was agreed with Paul et al. [10], which may be explained by the decreased functional activity of hepatocytes and innate immune system fatigue creating what was referred to as immune dysfunction related cirrhosis [11].

In this study, the ascitic fluid glucose level of SBP patients was significantly lower and this is in line with Lutz et al., [4] and Tsung et al.,[12], who reported that the ascitic level was lower in SBP patients since the concentration of ascetic glucose decreases dual to the bacterial consumption.

The current investigation has shown that the average value of SAAG in both SBP and non-SBP groups is $>1.1 \mathrm{~g} / \mathrm{dl}$ but without significant difference, which suggests that the ascite aetiology in these patients was portal hypertension. This is consistent with the findings of Agarwal et al[13], which indicated that the SAAG levels in all ascites was > 1.1g/dl, regardless of infection.

MiR-155 is one of the microRNAs (miRs) known to be short, non-coding nucleotide RNAs of around 21 to 25. MiRs work by regulating gene expression by binding to RNA, impacting vital biological processes. MiR-155 is the second most prevalent cell type observed in individuals with ascites and may affect liver inflammation and illness via its action as a macrophage activator[5].

MiR-155 has a major role in innate immunity, B cell maturation and activation of CD8+T cells[14].

MiR-155 seems to be crucial for the fibrotic process since, as discovered in fibrotic tissue, the loss of MiR-155 abrogates fibrosis when applied in vivo models, MiR-155 may be an early immune response suggesting acute infection and may be utilised as a marker of SBP [5].
The research analysed the difference in ascetic Micro RNA-155 levels of SBP and non-SBP patients showing greater levels in SBP patients than in Nabiel et al.[8], who said that ascite fluid levels are rising in SBP patients with MiR-155.

This findings is also in line with Lutz et al.[5] who said that SBP patients had greater amounts of Micro RNA-155 in their ascitic fluid than those of non-SBP. The invasion of microorganisms boosts the immune system. In SBP Micro RNA-155 ascites and serves as a powerful chemical factor for monocytes and lymphocytes stimulated by $\mathrm{T}$ [5].

In this research, the link between micro RNA-155 and TLC, polymorphs, MELD and child pugh score is not statistically significant in patients with SBP. No previous study has shown a link among SBP patients between micro RNA-155 and TLC, polymorphs, MELD and child pugh score.

In this work, the ROC curves analysis of MiR-155 as the SBP diagnostic marker showed that the sensitivity was 60 percent at the cut off level of 0,022 $\mathrm{CT}$, with a specimen area of 62 percent under the curve of 0,639 .

This is consistent with Lutz et al [5] who show increased levels of MiR-155 in SBP patients with 80 percent sensitivity and 90 percent specificities with 0.7 area.

In a research by Nabiel et al., [8] as a highly susceptible diagnostic marker with sensitivity $95.3 \%$ and $97.4 \%$ specificity in the diagnosis of SBP under the curve 0.95 were greater levels of ascitic MiR-155 in SBP patients.

In this study, the ROC curve analytics of PMN as an SBP diagnostic marker showed that the level of a decrease of 230 (103 percent) was 100 percent sensitive and that the SBP was 100 percent specific with an area of the curve 1 diagnosis, so that the PMN ascitic fluid figures are still the gold standard for diagnosis as in any directives such as Estekhri[15] which showed that PMN is the gold st mark for studies.

In the present studying, TLC and serum creatinine are a multivariant analysis of MiR-155 to considerably determine SBP positivity predictors, which is consistent with lutz et al.[5] which have shown that Mir-155 and serum creatins are considerably determining SBP positive predictors.

\section{Conclusion}

Although level MIR-155 was greater in ascetic fluid of SBP patients and is a predictor for development of SBP, it cannot nevertheless be employed as a diagnostic marker in SBP.

\section{References}

[1] B. A. Runyon, "Introduction to the revised American Association for the Study of Liver Diseases Practice Guideline management of adult patients with ascites due to cirrhosis 2012," Hepatology, vol. 57, pp. 1651-1653, 2013. 
[2] B. Niu, "Mortality from spontaneous bacterial peritonitis among hospitalized patients in the USA," Dig. Dis. Sci., vol. 63, pp. 1327-1333, 2018.

[3] H. Ling, M. Fabbri, and G. A. Calin, "MicroRNAs and other non-coding RNAs as targets for anticancer drug development," Nat. Rev. Drug Discov., vol. 12, pp. 847-865, 2013.

[4] P. Lutz, "MicroRNA-155 is upregulated in ascites in patients with spontaneous bacterial peritonitis," Sci. Rep., vol. 7, pp. 1-8, 2017.

[5] K. J. Fagan, "Ascites bacterial burden and immune cell profile are associated with poor clinical outcomes in the absence of overt infection," PLoS One, vol. 10, pp.e0120642, 2015.

[6] B. Yildirim, R. Sari, and N. Isci, "Patients with spontaneous bacterial peritonitis, and malignant and cirrhotic ascites.," J. Natl. Med. Assoc., vol. 97, pp. 276, 2005.

[7] J. A. Girón-González, "Serial analysis of serum and ascitic fluid levels of soluble adhesion molecules and chemokines in patients with spontaneous bacterial peritonitis," Clin. Exp. Immunol., vol. 123, pp. 56-61, 2001.

[8] Y. Nabiel, G. Barakat, and S. Abed, "Serum CD64 and ascitic fluid calprotectin and microrna-155 as potential biomarkers of spontaneous bacterial peritonitis," Eur. J. Gastroenterol. Hepatol., vol. 31, pp. 1064 1069, 2019.

[9] N. El-Toukhy and S. M. Emam, "Diagnostic and Prognostic Values of Monocyte Chemotactic
Protein-1 in Ascitic Fluid of Patients with Spontaneous Bacterial Peritonitis.," Egypt. J. Immunol., vol. 23, pp. 17-27, 2016.

[10] K. Paul, J. Kaur, and H. L. Kazal, "To study the incidence, predictive factors and clinical outcome of spontaneous bacterial peritonitis in patients of cirrhosis with ascites," J. Clin. diagnostic Res. JCDR, vol. 9, pp. OC09, 2015.

[11] A. Menshawy, "Safety and efficacy of rifaximin in prophylaxis of spontaneous bacterial peritonitis: a systematic review and metaanalysis," Curr. Drug Targets, vol. 20, pp.380 387, 2019.

[12]P. C. Tsung, "Predictive factors that influence the survival rates in liver cirrhosis patients with spontaneous bacterial peritonitis," Clin. Mol. Hepatol., vol. 19, pp. 131, 2013.

[13]M. P. Agarwal, B. R. Choudhury, B. D. Banerjee, and A. Kumar, "Ascitic fluid examination for diagnosis of spontaneous bacterial peritonitisin cirrhotic ascites," Journal, Indian Acad. Clin. Med., vol. 9, pp. 29-32, 2008.

[14]E. F. Lind, A. R. Elford, and P. S. Ohashi, "Micro-RNA 155 is required for optimal CD8+ $\mathrm{T}$ cell responses to acute viral and intracellular bacterial challenges," J. Immunol., vol. 190, pp. 1210-1216, 2013.

[15]R. Estakhri, L. Bartari, and M. Ghojazadeh, "Diagnostic value of serum procalcitonin level in the diagnosis of the spontaneous bacterial peritonitis," Immunopathol. Persa, vol. 6, pp. e19-e19, 2020. 